\title{
Transatlantica
}

Revue d'études américaines. American Studies Journal

\section{Ce que voir veut dire}

Compte rendu de l'exposition «Visions de l'Ouest : photographies de l'exploration américaine, 1860-1880». Musée d'Art Américain, Giverny, du 10 juillet au 31 octobre 2007. Commissaires d'exposition : François Brunet et Bronwyn Griffith

\section{Mathilde Arrivé}

\section{(2) OpenEdition}

Journals

Édition électronique

URL : http://journals.openedition.org/transatlantica/2373

DOI : 10.4000/transatlantica.2373

ISSN : $1765-2766$

Éditeur

AFEA

Référence électronique

Mathilde Arrivé, «Ce que voir veut dire », Transatlantica [En ligne], 2 | 2007, mis en ligne le 03 février 2008, consulté le 29 avril 2021. URL : http://journals.openedition.org/transatlantica/2373 ; DOI : https://doi.org/10.4000/transatlantica.2373

Ce document a été généré automatiquement le 29 avril 2021.

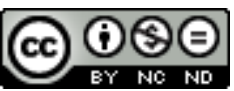

Transatlantica - Revue d'études américaines est mis à disposition selon les termes de la licence Creative Commons Attribution - Pas d'Utilisation Commerciale - Pas de Modification 4.0 International. 


\title{
Ce que voir veut dire
}

\author{
Compte rendu de l'exposition «Visions de l'Ouest : photographies de \\ l'exploration américaine, 1860-1880». Musée d'Art Américain, Giverny, \\ du 10 juillet au 31 octobre 2007. Commissaires d'exposition : François \\ Brunet et Bronwyn Griffith
}

\section{Mathilde Arrivé}

1 La photographie américaine a eu le vent en poupe en 2007. Les expositions consacrées à Diane Arbus, Lee Friedlander, Joel Meyerowitz, William Eggleston et Weegee lui ont donné un espace toujours plus légitime dans le musée français. Après Paul Strand, c'est au tour des photographies de l'Ouest américain des Great Surveys d'investir jusqu'au 31 octobre les galeries bilingues du Musée d'art Américain de Giverny. Les couloirs de l'exposition sont tout en bleu et brun pour ces 120 œuvres en noir et blanc - portfolios, vues stéréoscopiques et tirages originaux sélectionnés par François Brunet et Bronwyn Griffith parmi les milliers de vues réalisées dans le cadre des quatre missions officielles d'exploration de l'Ouest américain entre 1867 et $1879^{1}$ conduites, respectivement, par Clarence King, George M. Wheeler, Ferdinand V. Hayden et John W. Powell.

Comme le public victorien de l'époque (qui aime à «voyager dans son fauteuil » en parcourant des yeux un album photographique), on se réjouira de pouvoir redécouvrir à l'occasion de cette exposition ces splendides vues des Rocheuses, de la vallée du Yosemite, de Yellowstone et des canyons de l'Arizona.

3 A la croisée de l'histoire et du mythe, la photographie des Surveys est tout à la fois familière au public français, car relayée par le genre du Western, et méconnue dans la singularité de ses enjeux culturels, sociaux et médiatiques. Si cette photographie exerce un attrait pour le grand public dans ce qu'elle a d'iconique et d'apparemment immédiatement lisible, elle demeure un objet complexe, surprenant et rétif au consensus qui appelle, pour être compris, un effort de contextualisation.

Les photographies en contexte

4 Si la photographie des expéditions a déjà fait l'objet de plusieurs expositions outreAtlantique $^{2}$, c'est un événement tout à fait inédit en France. On a tant insisté sur l'américanité des photographies des Surveys que leur lecture s'est souvent déployée selon des problématiques strictement nationales faisant fi du contexte international 
qui fut celui de leur diffusion et de leur réception à la fin du $19^{\text {ième }}$ siècle. C'est donc la vie de ces images en dehors des Etats-Unis que les commissaires de l'exposition entendent à la fois rappeler, réactiver mais également exploiter, puisque l'intégralité du matériau photographique provient des collections françaises (associations, collectionneurs particuliers, musées et institutions ${ }^{3}$ ) qui furent souvent les destinataires et dépositaires de nombre de ces tirages. Des photographies furent en effet données par des agences américaines alors engagées dans une véritable " campagne de communication ${ }^{4}$, motivée par une recherche de prestige scientifique à l'extérieur du territoire national, mais également stimulée par les rivalités entre les différents Surveys et ministères en charge des projets.

5 Cette exposition se distingue donc par le souci des commissaires de documenter « les usages sociaux, politiques et médiatiques $»^{5}$ des photographies - autant de pratiques des images qui en éclairent largement le sens. Car il ne s'agit plus de situer seulement ces photos dans le strict champ de l'histoire de l'art ou de l'histoire des techniques, mais de les ancrer dans celui, plus englobant, de l'histoire culturelle en rendant compte (grâce à des documents d'archive et des textes de cadrage) de leurs conditions d'émergence, de production, de publication et de diffusion.

6 C'est ainsi que les épreuves grand format côtoient les formes dérivées destinées à la publication et à la commercialisation : gravures, estampes, vues stéréoscopiques, séries sur catalogue, accompagnées de texte et intégrées à divers supports (albums, brochures, catalogues des expéditions) selon les usages et les publics auxquels elles s'adressent. On se rend compte alors combien l'objet qui nous occupe est complexe tant il varie dans ses formes même. Un livret publicitaire en trois langues (allemand, français, anglais) témoigne de l'effort de diffusion des «Vues photographiques de l'Amérique » (issues de la mission Wheeler) à l'étranger. Pour ce qui est de la diffusion de ces photos en France, l'on peut voir un exemplaire de 1874 du «Tour du Monde $»^{6}$ qui servit de support, sous la forme de reportage illustré, à certaines photographies issues de la mission Hayden.

7 Car il s'agit bien alors pour le gouvernement américain de diffuser auprès d'un public peu averti (Américains citadins et de l'Est, Européens,) une imagerie de l'Ouest dans un contexte, rappelons-le, de prolifération tous azimuts de l'imprimé et de l'explosion des moyens de reproduction et des circuits de circulation. Car si les photos des expéditions ornent aujourd'hui les cimaises du MAAG, il ne faut pas oublier que dans la seconde moitié du $19^{\text {ième }}$ siècle ce corpus avait avant tout vocation à l'illustration.

L'Ouest des photographies : Pays, paysages

8 Ces photographies, conçues comme auxiliaires de l'incorporation de l'Ouest, auraient dû en toute logique être de stricts relevés topographiques et géologiques (ou ethnographiques, dans le cas des portraits), des artefacts à fort quotient scientifique participant concrètement à l'entreprise de normalisation de l'Ouest. Pourtant, ces photos ont une fonctionnalité quasi nulle, si ce n'est bien sûr leur efficacité communicationnelle, médiatique et symbolique. Car c'est bien dans la nature, constituée en paysage, que va se cristalliser aux lendemains de la guerre de Sécession le désir d'identité et d'unité et la recherche de distinction nationale. Tout se passe donc comme si, dans cet âge du Toc, la nature fonctionnait en bien culturel, sorte de plusvalue dans le concert des nationalismes, dont l'efficacité symbolique tend presque à éclipser la valeur marchande ou didactique. L'adéquation est alors maximale entre l'acte photographique et le geste culturel opéré par les Surveys - vastes projets 
d'archivage, de spectacularisation et de patrimonialisation du territoire national. La photographie, imaginée comme un prélèvement de réel, se fit en effet agent privilégié du projet puisqu'à chaque prise elle semblait bien «créer » du patrimoine, en produire des unités discrètes et manipulables. Preuve, trace, emblème ou substitut de l'objet figuré, la photographie en autorise une appropriation symbolique, qui se déploie visuellement de manière réglée et codée.

Topoï en devenir

9 La photographie des Surveys contribue à la formalisation de ce qui deviendra plus tard de véritables topoï de l'iconographie paysagiste américaine. Il s'agit de monumentaliser la nature de l'Ouest tout en minimisant son potentiel hostile: le paysage doit être grandiose mais accueillant, majestueux et hospitalier. Car la sauvagerie de la wilderness n'est promotionnelle que lorsqu'elle est savamment esthétisée, allégorisée, tenue à bonne distance. Il s'agit donc de créer un sublime pittoresque, une synthèse typiquement américaine (et pratiquée parallèlement par les peintres de la Rocky Mountain School) seule capable de susciter une défiance sereine face à la nature. C'est ainsi que, malgré l'aridité notable des régions visitées, les photos des Surveys sont semées de points d'eau, sources, cascades, torrents et lacs, que le temps long de l'exposition du wet-glass process traduit de manière veloutée et cotonneuse, presque voluptueuse, comme dans la "Cascade, Nevada Falls» de Carleton E. Watkins, prise vers 1861. L'environnement hostile de la wilderness, s'il fut agent d'édification morale et de régénération spirituelle pour les Puritains, devient recreational, lieu de divertissement et de jeu, objet de curiosité. On pensera aux « Wonders of Nature » de William Henry Jackson qui photographie les bizarreries de Yellowstone, son geyser ("Old Faithful », 1870) ou ses sources chaudes « Mammoth» (1871). Si les paysages de l'Ouest s'apparentent parfois à de vastes terrains de jeu, l'acte photographique luimême devient un acte ludique, un jeu sur les codes et les filtres, sur les détours de l'imagination analogique et de ses projections. Que l'approche soit picturale (surtout chez Jackson), architecturale ("Cathedral Mountain» de Carleton Watkins en 1865) ou culinaire (l'appétissante «Chocolate Butte» de William Bell en 1872), l'imaginaire est souvent anthropocentrique.

Terres vierges, espaces disponibles

Si l'Ouest, qu'il faut comprendre comme un vaste autoportrait, n'est pas terre d'abondance, il est plus que jamais une promesse, un lieu de possibilité. L'espace photographié reste absolument ouvert et non-circonscrit; les lignes s'enfuient hors champ et débordent le cadre; la perspective atmosphérique suggère toujours de lointains ailleurs. C'est le scénario pionnier de la découverte qui est réactivé sans que l'optimisme de l'exploration ne soit encore entamé par l'imagination désenchantée, nostalgique et mélancolique qui imprègne certaines photographies du début du $20^{\mathrm{ième}}$ siècle. L'accent est donc mis sur l'ouverture et la disponibilité (au regard), le photographe étant toujours positionné à l'orée d'un paysage, au seuil d'une vue. A l'instar de "L'entrée du Grand Canyon » de Timothy O'Sullivan (1872), la photographie de l'Ouest est une invitation au voyage et au tourisme alors que le chemin de fer et la route pénètrent toujours plus avant dans ces territoires qui semblent rester imperturbables - pôles d'équilibre et de stabilité - face à l'industrialisation et l'urbanisation massive du pays'. Seuls quelques clichés réalisés par Jackson pour le compte de l'Union Pacific Railroad témoignent de l'avancée de la voie ferrée, envers (ou revers) machinique du rêve pastoral. Le cheval de fer, drainant touristes et capitaux 
dans les terres de l'Ouest, est un adjuvant à double tranchant qui rend possible l'exploration et la valorisation de l'Ouest tout en compromettant ce qui en constitue l'attrait, le fantasme de sa «virginité ». A cet égard, les photographes des Surveys font bien figure de proto-touristes, premiers recreationists et ancêtres de cette leisure class qui, munie d'un Kodak, viendra visiter l'Ouest quelques décennies plus tard. Déjà, T. O'Sullivan, W. H. Jackson, William Bell, J. K. Hillers et les autres laissent des indices de leur présence : bouteille vide, matériel photographique, empreintes, traces de pas... une façon de marquer l'environnement, d'imprimer un passage, de notifier une présence - une variation sur l'art de la signature en photographie.

Regards

11 Que l'on parle de views, de vistas, de scenes ou de visions, l'œil est une métaphore centrale dans l'Amérique du $19^{\text {ième }}$ siècle. La machine photographique, produit d'une modernité démiurgique, en est l'extension et l'emblème agissant.

Empaysagement ${ }^{8}$

12 Certaines lectures veulent voir dans les photos des expéditions la littéralisation de l'idéologie expansionniste américaine et de l'esprit de conquête de la fin du $19^{\text {ième }}$ siècle. Certes, dans la plupart des tirages, le «je » voyant américain embrasse de son regard panoptique la vue qu'il surplombe. C'est là une composante structurale récurrente qui, si elle trahit des velléités de contrôle et d'appropriation, s'inscrit également dans une recherche d'effets et procède d'une adaptation aux contraintes du paysage - son immensité oblige à la distance et au surplomb. « Photographing in High Places » (1869) de W. H. Jackson, une vue d'en haut, affiche explicitement cette posture. Conformément aux conventions du sublime, la photographie des Surveys privilégie des échelles grandioses, amplifiées par le contraste entre le très grand et le très petit, mettant l'accent sur la masse du paysage plutôt que sur ses détails. Symptomatiquement, la photographie des Surveys est une pratique de l'image qui ignore absolument la vue en gros plan.

13 Et pourtant, conclure au regard hégémonique reviendrait à ne pas prendre en compte ces représentants minuscules de l'observation intégrés au paysage, presque invisibles tant ils se fondent et s'oublient dans son ampleur, comme dans les vues du «Grand Canyon du Colorado (Arizona)» de Jackson ou de "Chocolate Butte» de Bell. Ces figures d'observateurs constituent certes un élément fonctionnel indicateur d'échelle mais n'en demeurent pas moins miniaturisées par l'immensité du paysage. Dans les photos des Surveys, est souvent à l'œuvre une double posture regardante: celle, invisible et surplombante du photographe "in high places »; l'autre, intégrée à la scène, écrasée par la majesté des canyons. L'inscription de l'observateur dans la nature est dialectisée, puisque le "magisterial gaze » côtoie dans la même photo son double révérenciel, son contrepoint révérencieux ${ }^{9}$.

W.H. Jackson, « Grand Canyon du Colorado »

http://americanart.si.edu/images/1994/1994.91.85_1b.jpg

14 A travers l'ambivalence de ce regard sur la nature, c'est l'ambivalence de la conquête (entre gain et perte, maîtrise et impuissance) qui est suggérée dans une photographie oscillant visuellement entre nécessité de distance et désir de proximité. William Bell, dans sa «Vue du Grand Canyon en regardant vers le sud (Colorado River, Shivwits Crossing)» de 1872 évoque jusqu'au vertige les possibilités et les limites de 
l'exploration, dans une vue plongée dont il exalte le potentiel (plastique) mais reconnaît les dangers.

William Bell, « Vue du Grand Canyon en regardant vers le sud (Colorado River, Shivwits Crossing) » de 1872

http://americanart.si.edu/helios/AmericanPhotographs/images/bellw04b.jpg

Ce que racontent les photographies des Surveys, c'est bien l'histoire de la rencontre de l'homme et du paysage - une rencontre, voire une relation, dont elles déclinent les modalités sur le mode sérieux de la découverte et de la conquête ou sur le mode picaresque ou humoristique de l'anecdote.

Miroirs

16 Si le but avoué et officiel du projet photographique des Surveys est bien d'aider à incorporer l'Ouest au territoire national, à l'imaginaire national et à la somme des visibilités américaines, le but individuel et intime de chaque photographe est de s'amuser des possibilités de son propre regard. Cette articulation entre la dimension collective et individuelle de la photographie de l'Ouest s'opère donc autour de l'acte de voir et du travail du regard, comme en témoigne l'inclusion répétée des figures de l'observation. La composante réflexive est également tissée à travers le trope traditionnel du miroir d'eau réfléchissant et déformant dans " Mystic Lake (Montana)» de W. H. Jackson, dans « La tour en reflet (Rio Virgen, Utah)» de John K. Hillers (1870) ou dans cette vue d'El Capitan de Carleton Watkins.

C. E. Watkins, « El Capitan, Yosemite» (1860)

http://www.nga.gov/exhibitions/2000/watkins/images/elcapitan_390.jpg

Entre miroir (objectif) et prisme (subjectif), l'image photographique a encore un statut incertain au $19^{\text {gème }}$ siècle. On est surpris par la recherche proprement formelle et plastique d'un O'Sullivan dans ses "Dômes de tuf, Pyramid Lake, Nevada » de 1867-1868, où les formations rocheuses se dissolvent dans la brume en volutes biomorphiques presque abstraites. Par un travail sur la texture et de savants effets de matières, formes et couleurs résistent à toute référentialité, que seul le titre de la photo rappelle. La mimésis est ici éprouvée comme à rebours des vertus censément « signalétiques » de la photographie.

T. O'Sullivan, « Dômes de tuf, Pyramid Lake, Nevada », 1867-1868, http://americanart.si.edu/images/ 1994/1994.91.142_1b.jpg

18 On a dit de l'Ouest des photographies des Surveys qu'il était pittoresque, avec ses lacs, rivières et étangs. Mais si ce motif récurrent interroge les pouvoirs de la mimésis, il mobilise également la lumière et ses variations. Sculpter les vues en enregistrant les effets modelants de la lumière sur l'eau ou la roche, c'est reconnaitre la plasticité du paysage. Pour le photographe, c'est affirmer son inventivité dans une exploration, cette fois formelle, de la nature. Timothy O'Sullivan revendique ainsi cette démarche esthétique dans son "Ombre et lumière au Black Canyon, vue depuis Mirror Bar » de 1871.

Le travail du temps

19 Si les photographes, à l'instar de leurs prédécesseurs peintres, exploitent les ressources plastiques de la lumière, ils en explorent également les pouvoirs dramatiques et dynamiques, déclinant ainsi les différents visages d'un même paysage lorsque sont mis 
en séquence plusieurs clichés pris à différentes heures de la journée. On pense aux séries que fit O'Sullivan du Grand Canyon, du Black Canyon ou des Shoshone Falls.

T. O'Sullivan, «Black Canyon »

http://www.moma.org/images/collection/FullSizes/50013074.jpg

Intégrer le paysage dans une séquence c'est tenter d'inscrire des entités géologiques apparemment immémoriales et immuables dans le temps chronologique et linéaire de l'histoire. Entre permanence et promesse de transformation, la nature semble rattrapée par la possibilité d'un devenir, voire d'une érosion. Dans ce contexte, la photographie du «Roc taillé par les vents de sable » (1871) de O'Sullivan est le constat paradoxal de la fragilité et de l'irréductibilité de la nature ${ }^{10}$.

Et, si comme on l'a dit, l'objet de la photographie est la lumière, c'est sûrement aussi qu'en dernière analyse, son véritable objet est le temps, son passage et ses effets ${ }^{11}$. A cet égard, la photographie des Surveys réfracte deux impulsions contradictoires, celle de monumentaliser un paysage naturel grandiose en l'inscrivant dans le temps du mythe (on sera dans le registre sublime) et celle de rendre accessible un paysage rattrapé par le temps de la modernité (on sera dans le registre pittoresque). Dans ce dernier cas, il s'agira d'incorporer le paysage à une narration, à la petite histoire, celle des acteurs de l'exploration.

On découvre d'ailleurs d'étranges natures mortes: ici une bouteille vide, là une embarcation, pour un jeu de piste qui, bien qu'il soit dénué de la gravité du memento mori, dissémine cependant les traces éphémères d'un passage, comme dans les célèbres "Dunes de sable» de O'Sullivan (photographie emblématique qui fait l'affiche de l'exposition et la couverture du catalogue). Entre effacement et inscription, la présence/absence humaine est toujours enregistrée, souvent après coup, tandis que les photographes jouent, semble-t-il, à se faire disparaître, pour rire de cette disparition et s'en rendre maître, pour en exorciser l'arbitraire en la faisant apparaître.

T. O'Sullivan, « Dunes de sables » (Carson Desert, Nevada, 1868)

http://www.archives.gov/research/american-west/images/008.jpg

Images d'Indiens

Tel est le titre de la section consacrée aux portraits d'Amérindiens pris dans le cadre des Surveys. C'est l'aspect le plus saillant, le plus distinctif et peut-être le plus ambitieux de cette exposition qui, dans un souci de rendre compte de la totalité du matériau photographique des missions d'exploration, a choisi d'intégrer pour la première fois "les images d'Indiens"-certes très minoritaires parmi les milliers issues des Surveys - et de faire voisiner visages et paysages. Il faut noter qu'originellement, photos de paysages et photos d'Indiens ont toujours été publiées séparément, dans des volumes bien distincts. D'ailleurs, avec ces portraits, d'autres photographes s'illustrent comme Antonio Zeno Shindler et Alexander Gardner, même si William H. Jackson et John K. Hillers ont également collecté des portraits indiens pour leurs missions respectives.

Est-ce à dire que ces deux catégories d'images poursuivent des logiques différentes, donnent lieu à des pratiques, des usages et des lectures différentes ? Bien évidemment. Et si le but de l'exposition est de réunir en son sein l'intégralité du corpus, reste néanmoins une difficulté d'articulation entre paysages et visages, inhérente au projet des Surveys, qui semble reproduite dans l'agencement scénographique et le dispositif visuel et textuel de l'exposition. Les organisateurs ont en effet préféré un agencement 
thématique par sujets («Grands espaces"; «Eléments du paysage »; «Timothy H. O'Sullivan »; «Images d'Indiens : portraits et habitats ») à une organisation par Surveys, trop chronologique, trop technique, trop opaque. Disposés sur un pan de mur transversal aux allées où figurent les paysages, les portraits s'intègrent tout en se distinguant. A dire vrai, c'est souvent sur eux que les visiteurs s'attardent le plus longuement. Car en effet, on ne peut qu'être interpellé par cette fracture, si manifeste, entre ces visages et ces paysages impersonnels de pierre et de ciel - deux ensembles d'images qui ont en commun la même frontalité mais dont on a du mal à croire qu'ils procèdent d'une même impulsion tant ils invitent à des réceptions différentes. L'intérêt réside dans cette fracture (une fracture que même dans le présent commentaire il est malaisé de ne pas reproduire).

La majorité de ces clichés furent réalisés en studio dans la lignée des portraits de délégation, sur fond absolument neutre, dans un décrochage avec le cadre spatiotemporel de la prise photographique, dans une évacuation de tout contexte et de toute relation, que l'on peut comprendre comme une tentative de neutraliser tout affect. Les Indiens sont en effet déplacés physiquement de leur environnement vers l'espace du studio tandis qu'ils le sont symboliquement dans l'espace de la photographie et du livre. Rien ne vient rappeler leur vie dans les terres «vierges» de l'exploration : ces photos sont des hypostases, elles traduisent le refus d'imaginer le lien entre ces populations et le territoire exploré. A travers l'assignation dans "des musées de papier ", l'acte photographique est à la fois une métaphore et une littéralisation de la relégation à la marge des populations indiennes. On notera cependant que quelques très rares photographies in situ, telle que « Vue sur Apache Lake dans la Sierra Blanca; Deux éclaireurs Apaches au premier plan » (1873) de O'Sullivan réfractent des relations, mais sur le mode de l'hostilité. La présence indienne y est en effet figurée comme une menace à écarter, un obstacle à... déplacer.

http://americanart.si.edu/images/1994/1994.91.143_1b.jpg

Cette exception mise à part, le but de ces « images d'Indiens » n'était pas d'évoquer les rapports sociaux de l'Ouest ou les "guerres indiennes " qui faisaient rage dans les années 1870 et que ces galeries de portraits pourtant laissent entendre tant elles s'évertuent à ne pas les montrer. Bien que les Amérindiens soient des acteurs majeurs de la vie de l'Ouest, ils ont dans ces clichés des allures d'ancêtres depuis longtemps disparus, tant prévaut alors le trope multiforme du Vanishing Indian. Malgré les courtes biographies qui accompagnent les portraits et témoignent du rôle des Indiens en tant qu'agents historiques, les photographies présentent des figures presque cataleptiques et comme minéralisées, aux allures spectrales et sculpturales quand la lumière miroite sur les étoffes et la peau. "Ce sont des images beaucoup moins exploratoires que testamentaires " peut-on lire dans le catalogue de l'exposition ${ }^{12}$. Les temps sont à la fabrication d'une mémoire.

On l'a dit, les relations sont gommées derrière une posture photographique d'archivage systématique - une apparente scientificité qui confère légitimité aux photographes d'alors. D'une certaine manière, on pourrait avancer que le portrait fait au visage ce que le paysage fait à la nature : il le "patrimonialise ", le normalise et le soumet à des codes. C'est ainsi qu'aux conventions du portrait de délégation se superposent parfois celles du portrait anthropométrique, que l'on retrouve dans le dispositif face-profil utilisé par Alexander Gardner dans ses photos de Long Pied, chef Sioux Yankton, (1867). 
Et pourtant quelque chose dans ces photos nous « point ${ }^{13}$ : l'éloquence silencieuse du corps et du visage dit le malaise face à l'appareil photographique, la résistance à laisser voir et à céder à l'entreprise d'absolue mise à vue et de classement encyclopédique de la population amérindienne. En témoigne le «Type indien » (1860-1869) d'Antonio Zeno Shindler qui, au rebours de son titre, donne à voir un bougé-flou formel et indistinction visuelle - qui vient perturber le protocole de la pose et ses velléités d'imposition. Ce bougé, symptôme d'une individualité imprévisible, dynamique et irréductible, est d'autant plus signifiant que le titre («type indien ») essaie quant à lui de ramener le regardeur à une lecture générique et typifiante, d'autant plus vaine et arbitraire qu'elle est ostensiblement contredite par l'image. L'intérêt repose dans l'écart entre ce que la photographie veut dire et ce qu'elle laisse voir.

29 A ces portraits en studio s'ajoutent quelques photos in situ, beaucoup moins nombreuses. Si le lien entre population et territoire est toujours évacué, en revanche la confusion de la personne indienne et du milieu naturel est exacerbée : par le biais d'un continuum formel entre figures humaines et éléments du paysage, les Indiens sont imaginés comme des extensions du monde naturel. Dans cette tentative de naturaliser la « sauvagerie » autochtone (une autre manière de justifier la conquête), le propos est souvent eurocentrique et racialiste. A cet égard, comme cela est expliqué dans le dossier de presse, il existe des orientations différentes selon les Surveys : tandis que la mission Wheeler souligne une hostilité potentielle, la mission Powell se veut plus "progressive ». On voit mise en scène une "compassion» souvent paternaliste et voyeuriste, à l'œuvre par exemple dans « Guides Mojaves assoupis, Arizona (1871) » de Timothy O'Sullivan (mission Wheeler).

« Guides Mojaves assoupis, Arizona (1871)» de Timothy O'Sullivan.

http://sunsite.berkeley.edu/FindingAids/dynaweb/calher/usgswest/figures/I0045613A.jpg

30 Dans cette photo est suggérée une relation scopique unilatérale qui se traduit dans les postures corporelles et se déploie dans l'espace de la photographie à travers la verticalité du regard blanc et la non-réciprocité de l'observation. S'énonce visuellement la hiérarchie entre l'« expert » américain, en posture assise et à l'étude, et les corps des deux scouts allongés à l'horizontale dans le sable.

« Ku-ra-tu, femme Paiute (1872)» par John K. Hiller

http://www.eastman.org/taschen/m198109670116.jpg

31 Dans le portrait de « Ku-ra-tu, femme Paiute (1872) » réalisé par John K. Hillers pour la mission Powell, la jeune femme semble soumise à un protocole de pose, placée devant une paroi rocheuse pour renforcer le lien entre son corps et le monde minéral. Et comme l'analyse très finement Mick Gidley dans le catalogue de l'exposition ${ }^{14}$, bien qu'elle se prête aux procédures de cette scénographie, ce n'est pas son appartenance innée au monde naturel qu'elle montre mais bien plutôt les signes de son inconfort face à cette mascarade ridicule. A travers la résistance de cette femme au scénario qu'on veut lui faire jouer, c'est tout le dispositif racialiste qui est dénaturalisé.

32 Avec ces «images d'Indiens" on a donc bien affaire à un corpus photographique exigeant qui demande relecture, à l'instar de ces pictogrammes précolombiens dans lesquels l'imaginaire orientaliste d'un William Bell voit des hiéroglyphes égyptiens (comme en témoigne le titre de sa vue : «Hieroglyphic Pass en face de Parowan, Utah » de 1872). Codes, écriture et mystères de l'altérité font achopper le regard et continuent invariablement de dérouter. Si la rencontre avec l'autre s'inaugure souvent sous le 
signe de la méprise, la photographie nous offre la possibilité de revenir aujourd'hui sur ses traces, de revisiter sa présence.

Conclusion

33 La photographie de l'exploration de l'Ouest américain donne lieu à plusieurs lectures, qu'elles soient culturaliste (aspects médiatiques des Surveys), esthétique (aspects formels des photos) ou théorique (aspect paradigmatique du corpus). La présente exposition n'invite pas à faire le choix entre l'une ou l'autre de ces lectures, mais encourage bien plutôt à les combiner. On navigue en effet entre la logique officielle, fédérale et collective des Surveys - envisagée dans sa "fonction de communication sociale» ${ }^{15}$ - et la logique individuelle de la pratique photographique singulière. La spécificité stylistique, technique ou communicationnelle de chaque photographe n'est jamais sacrifiée à la compréhension globale du projet. L'exposition dynamise la fine dialectique entre les dimensions politiques et poétiques, historiques et esthétiques qui travaillent le corpus en profondeur. C'est ainsi qu'en marge de la démarche de documentation et de spectacularisation du paysage américain, l'on peut découvrir de fines stratégies visuelles faisant intervenir le jeu, l'humour et la narrativité ${ }^{16}$, des composantes qui se tissent au fil des photos, des notes et des textes.

Que l'on parle de "picture makers", d'«illustrateurs", d'«opérateurs", ou de «techniciens ", les photographes des Surveys, au statut encore incertain à la fin du 19 ième siècle, n'en demeurent pas moins « les primitifs de la photo américaine ». Qu'est-ce à dire, sinon qu'avec les Surveys et grâce au concours d'un véritable "mécénat d'état " fait jour un corpus qui aura bientôt valeur de matrice? La photographie des Surveys, dans les mots du commissaire de l'exposition et spécialiste François Brunet, est « une création collective qui devait s'imposer d'abord comme la matrice des westerns et une mémoire visuelle de l'Ouest, avant de devenir le corpus maître de son âge classique ${ }^{17}$.

\section{NOTES}

1. Si la chronologie des quatre expéditions fournit à l'exposition son cadre temporel, certaines photographies ne s'y inscrivent pas strictement. C'est le cas par exemple des photos de Watkins, prises avant les expéditions dans les années 1860, et de certaines photos de William H. Jackson et de John K. Hillers prises après 1880.

2. La première exposition, «Era of Exploration » eut lieu en 1975 au MET ; voir Weston J. Naef et James N. Wood, Era of Exploration, The Rise of Landscape Photography in the American West, 1860-1885 (New York : Metropolitan Museum, 1975). La dernière en date, "One/Many ", fut organisée par Joel Snyder en 2006 à Chicago, mettant l'accent sur les panoramas et les séries photographiques : voir Joel Snyder et Josh Ellenbogen, One/ Many: Western American Survey Photographs by Bell and O'Sullivan (Chicago : Smart Museum of Art, The University of Chicago Press, 2006).

3. Si la plupart des tirages et objets présentés proviennent de la Société de Géographie, de la BNF et du Musée du Quai Branly, d'autres sont issus des collections du Musée d'Orsay, du Musée Nicéphore Niépce à Châlon-sur-Saône, de la Bibliothèque 
Administrative de la Ville de Paris, de la Bibliothèque Centrale du Muséum National d'Histoire Naturelle, du Club Alpin français, de l'Ecole Normale Supérieure et de la collection Marc Walter. On notera que cette exposition est organisée avec le soutien de la Société de géographie et de la Bibliothèque Nationale de France.

4. François Brunet, « Politiques photographiques de l'exploration américaine », dans Visions de l'Ouest, photographies de l'exploration américaine, 1860-1880. Catalogue de l'exposition (Musée d'art américain Giverny, Terra Foundation for American Art, 2007), 29.

5. Brunet, « Politiques photographiques », 25.

6. « Nouveau journal des voyages $» n^{\circ} 28$, Hachette, 1874.

7. L'absence de «traces » du chemin de fer est d'autant plus notable que le Pacific Railroad Act fut signé en 1862, suivi de près par le Homestead Act (1863), qui inaugure le peuplement des terres « vierges » de l'Ouest. Mais les compagnies de chemin de fer avaient leurs propres photographes.

8. $\mathrm{Si}$, comme on l'a dit, l'Ouest des photographies est souvent vierge, il est en revanche rarement vide. De photo en photo, la présence humaine pose la question de l'inscription du corps dans le paysage, un corps américain (plutôt qu'indien) et blanc.

9. A. Boime, The Magisterial Gaze. Manifest Destiny and the American Landscape Painting (Washington et Londres : Smithsonian Institution Press. 1991), 188. A. Boime parle également de «the reverential gaze».

10. Faut-il rappeler que c'est parallèlement au projet des Surveys que sont initiées dans les années 1870 les premières politiques de conservation avec la création du Parc National de Yellowstone en 1872 ?

11. Finalement, cette collecte de quelques milliers de vues dans le cadre des Surveys a bien des allures de collection si bien que, considéré dans son ensemble, le corpus photographique des Surveys s'apparenterait à une vaste série qui, en tant que telle et parce qu'elle est discontinue, parle du temps, signifie le temps, en est le signifiant.

12. Brunet, « Politiques photographiques », 99.

13. Quelque chose nous point ou nous pique, à l'instar du « punctum » dont parle Roland Barthes dans La chambre claire (1980, in Euvres complètes $V, 1993,809)$ pour évoquer les pouvoirs d'interpellation de certaines photographies.

14. Voir Mick Gidley, «'Out West and In the Studio', Official Photographs of Indians during the Great Survey Era ", dans Visions de l'Ouest, 33-45.

15. Brunet, « Politiques photographiques », 29.

16. Humour et narrativité sont les deux aspects de la photographie de l'Ouest que François Brunet s'attache à restituer en traçant d'insolites parcours de sens qui se déploient en parallèle de la logique officielle des Surveys. Voir « Revisiting the Enigmas of Timothy O'Sullivan : Notes on the William Ashburner Collection of King Survey Photographs at the Bancroft Library ", History of Photography $31: 2$ (été 2007).

17. Brunet, « Politiques photographiques », 12 et 29. 
INDEX

Thèmes : Trans'Arts

\section{AUTEUR}

MATHILDE ARRIVÉ

Université Michel de Montaigne Bordeaux 3 